\title{
SYSTEMATICS OF DEFECT STRUCTURES IN GLASSES WITH IONIC SUBSTITUTIONS
}

\author{
W. C. LEVENGOOD \\ The University of Michigan, Institute of Science and Technology, Ann Arbor, Michigan
}

(Received 4 September 1962; revised 31 December 1962)

\begin{abstract}
The effects of various ionic substitutions on defect structures in glass were investigated in soda-lime-silica systems. It is shown that detailed measurements of flaw characteristics provide a useful tool for investigating subtle internal energy variations in this solid. Influences of specific ions were quantitatively studied by determining the parameters of flaw length $\left(F_{1}\right)$, and flaw number $\left(F_{n}\right)$. Flaws produced on freshly broken surfaces under controlled loading conditions, were shown to be a direct indication of the stress response of the glass network. Relative bond strength and rigidity or brittleness of the structures varied with the ionic field strengths of added cations. Alkali ions in the form of oxides, were substituted for sodium and alkaline earth ions for calcium. Other additions and substitutional ions were also studied in detail. In general, the changes in flaw parameters were found to agree with theoretical predictions of internal energy changes based on the manner in which the ion entered the network, that is, as a lattice modifier, interstitial or network former. In specific systems, the variations in flaw length and flaw numbers were tentatively explained by changes in defect concentration and free volume as the ions were substituted.
\end{abstract}

\section{INTRODUCTION}

FolLOWING the discovery of a method for quantitatively studying minute structure-sensitive flaws in glass, (1) it became apparent that this technique could be utilized to study the effect of various ionic substitutions in the network. Detailed investigations have shown that these defects have properties similar to dislocations in crystals and that the stress energies required to move or form these flaws are of the same order of magnitude as the energies necessary to move dislocations in crystalline lattices. It has been repeatedly demonstrated that the minute flaws in glass are not the same as gross bond rupture or visible cracks and the structure-sensitive charactcristics of the defects have been confirmed in numerous investigations.

To a more limited degree, the influences of composition variations on the defect patterns have been quantitatively shown in soda-lime-silica systems. ${ }^{(2,3)}$ A dynamic spherical indenter technique was employed to create flaws under known, controlled loading conditions. The length of the flaws produced by this device was found to vary in a systematic manner with various ionic substitutions.
The results of more extensive experimentation pertaining to the influences of substitutional ions on defect properties are presented in this paper. Substitutions were made in three component systems, and two flaw parameters were studied; namely flaw length and number. By considering both of these parameters, the relative bond readjustment or yield was determined for a given stress condition. These quantitative flaw characteristics were compared with the ionic field strength or polarization of substituted ions. A hypothesis of changes in free volume with changes in internal ionic configuration was found to be in qualitative agreement with the experimental data. In general it is shown that the structure-sensitive defect patterns are related to internal energy variations.

\section{EXPERIMENTAL}

Oxide substitutions were made in a base glass containing 70 per cent silica and an initial sodalime ratio of $1.14\left(\mathrm{Na}_{2} \mathrm{O} / \mathrm{CaO}\right.$ on a weight per cent basis). The selection of this particular glass was not entirely arbitrary, as will be shown in a following Section. Reagent grade materials were used throughout these studies. The silica was 
pulverized (200 mesh) and was also of reagent grade. The basic raw materials were thoroughly mixed, placed in platinum-rhodium crucibles* and melted at $1400^{\circ} \mathrm{C}$ for $30 \mathrm{~min}$. Sufficient batch was used to give $20 \mathrm{~g}$ of melted glass, which was poured out into a sample about in. thick and $1 \frac{1}{2}$ in. in diameter. The samples were generally free of bubbles (at most only two or three "seeds") and the internal homogeneity appeared to be comparable to commercial glass. These samples were annealed with a final internal tension of around 20 p.s.i. (somewhat lower stress than in ordinary commercially annealed ware). After annealing, a test slug was diametrically scored with a glass cutter, then broken open using a fast break to give a relatively smooth test surface. The dynamic spherical indenter was applied within $30 \mathrm{sec}$ after breaking. This indenter device was previously described ${ }^{(3)}$ and consists of a $\frac{G}{1 \mathrm{~B}}$ in. steel ball provided with an axle so that it can rotate longitudinally under an applied vertical load. A $500 \mathrm{~g}$ load was used with a horizontal velocity of about $0.5 \mathrm{~cm}$ per sec. After making the ball trace, the samples were subsequently etched to develop the flaw patterns.

The flaw counts and flaw lengths were determined by microscopic examination using about $100 \times$ magnification. An ocular micrometer with a precision of about $\pm 0.001 \mathrm{~mm}$ was used to determine the flaw length. Only those flaws which were directly associated with the trace lines were included in the analyses. Under these loading conditions, at least 30 flaws were measured on all of the samples studied. The flaw length designated as $F_{l}$ is the average of length of the flaws extending from the trace line and the flaw number designated as $F_{n}$ is the average number of flaws nucleated per $\mathrm{mm}$ of trace line.

\section{INTERPRETATION OF FLAW CHARACTERISTICS}

The value of flaw length $\left(F_{1}\right)$ is taken as an indication of the relative bond strength under the given loading conditions. The greater the $F_{l}$ value,

* Materials corrosive to platinum were melted in porcelain crucibles. Impurities from these crucibles were found to produce a slight increase in the flaw parameters of the base glass. The use of porcelain did not, however, significantly alter the shapes of the flaw parameter vs. composition curves. the weaker the bonds; in other words, the flaws propagate outward from a high stress region until they reach a critical zone where the clastic strcss is no longer of sufficient magnitude to produce bond displacement or slip. Detailed studies have indicated ${ }^{(3)}$ that the flaw lines are the edges of slip planes created by mechanical or thermal stresses. The origin or core of the slip plane lies below the surface $(\sim 12 \mu)$. The linear magnitude of the slip line is closely related to the internal structure of the glass. This situation has been defined by GraY.(4) "In theory all internal imperfections occasioned by impurities, structural defects or energy changes will have related effects at the surface, modifying the surface activity."

The number $\left(F_{n}\right)$ of flaws produced with a given applied stress is an indication of the bond rigidity and might be loosely termed a "brittleness factor". In a flexible network, the stress energy is dissipated by a greater bending or distortion of bonds and a few flaws are initiated. A more rigid structure nuclcates more flaws, due to the fact that bonds will break before much bending occurs. The parameter of flaw number might be expected to be related to Young's modulus, and this possibility is indicated in a later Section.

If we desire to obtain information regarding the relative number of bonds broken, or the degree of slip under a given stress, it is necessary to consider the product of flaw length $\left(F_{l}\right)$ and flaw number $\left(F_{n}\right)$. This product is designated simply as

$$
N_{b}=\left(F_{l}\right)\left(F_{n}\right)
$$

This empirical relationship, as previously mentioned, has already been critically examined and it has been shown that the parameters vary with type and composition of the glass. The relationship between stress and radial distance $r$ from the central point of loading on the spherical indenter is given by

$$
\sigma=\left[\frac{(1-2 \nu)}{2 \pi}\right] P / r^{2}
$$

where $P$ is the applied load and $\nu$ is Poisson's ratio. The critical yield stress occurs at the point where the flaws, initiated at the trace line, terminate. This stress may be found by substituting $F_{l}$ for $r$ in equation (2) which gives

$$
\sigma_{\mathrm{crit}}=P K /\left(F_{1}\right)^{2}
$$


where

$$
K=(1-2 \nu) / 2 \pi
$$

If we assume that the glass is isotropically uniform then $\sigma_{\text {crit }}$ is a constant determined by the ratio of applied load $P$ to flaw length squared, providing, of course, the load is high enough to exceed this critical stress and initiate flaws. The load, therefore, determines in a unique way the distance the flaw will spread before the shear forces have been attenuated to a level where they are counterbalanced by the cohesive or bond energy of the glass. By rearrangement of equations (3) and (4) we may therefore predict morc precisely how the flaw length is related to the applied load by

$$
F_{l}=\left(P K / \sigma_{\mathrm{crit}}\right)^{1 / 2}
$$

Equation (5) shows that one would expect a linear relationship between the flaw length and the square root of the applied load. The data presented in Fig. 1 were obtained with a commercial sheet glass and show that this relationship is confirmed over a wide range of applied loads. Each point on this curve represents an average of over 50 flaw measurements. The form of the equation is, however,

$$
F_{l}=a P^{1 / 2}+b
$$

The constant $b$ in equation (6) represents a threshold stress which must be introduced into the system to initiate the flaws. The form of the curve in Fig. 1 has been confirmed with different types of glasses and these data provide additional evidence that the flaws associated with the trace line are a direct indication of the response of the network to applied stress.

\section{NETWORK SUBSTITUTIONS}

Before making substitutions, using the socalled network modifying ions, systematic variations were made to determine the manner in which the flaw characteristics change in three component systems. Three soda-lime-silica systems were made up for this study, and in each series the silica was held constant at 60,65 and 70 per cent with the soda-lime ratio (based on weight percent) varied over wide limits. The results of the flaw length measurements are summarized in Fig. 2 (the 60 per cent $\mathrm{SiO}_{2}$ curve was previously given(2) but is redrawn here for comparison). It may be seen that the flaw lengths generally increase with increasing silica content of the glass. In each series, there appears to be a maximum in the curve, and the significance of this will be discussed later.

In spite of the flaw length increase with increasing silica, one would expect that with increasing concentration of this network-forming oxide, a general increase in the strength of the structure

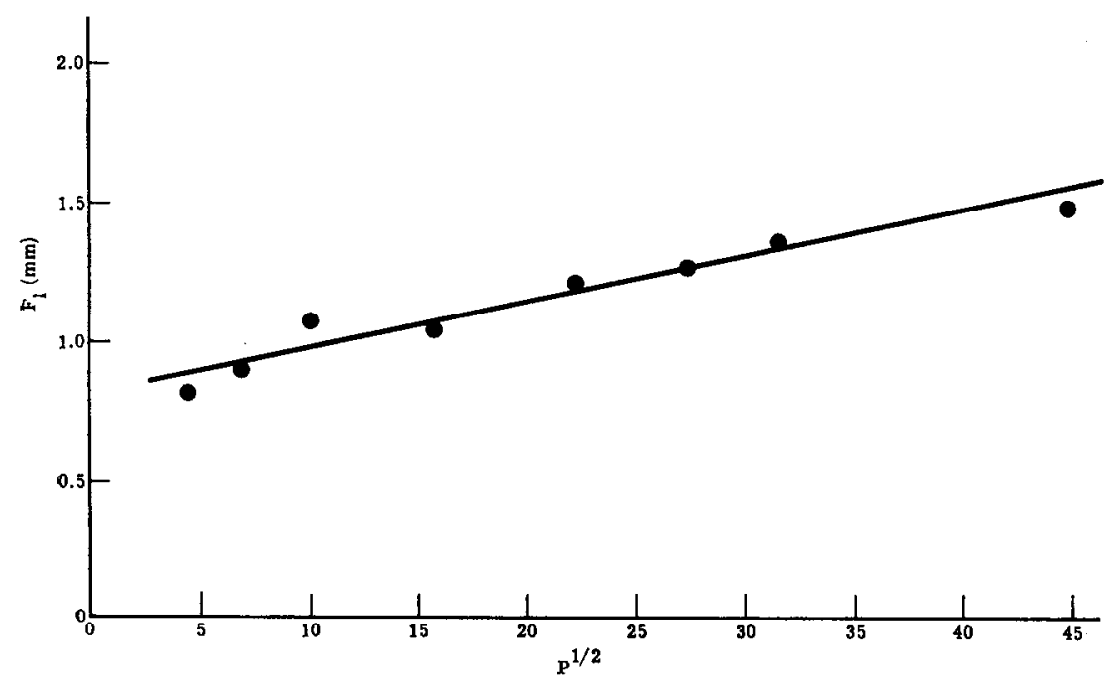

FIG. 1. Relationship between load $(P)$ and flaw length $\left(F_{l}\right)$. Indenter applied to the fresh surface of a commercial sheet glass. 
or a decrease in the $N_{b}$ product would occur. That this is generally true may be seen in the data representing flaw number and $N_{b}$ product in Table 1.
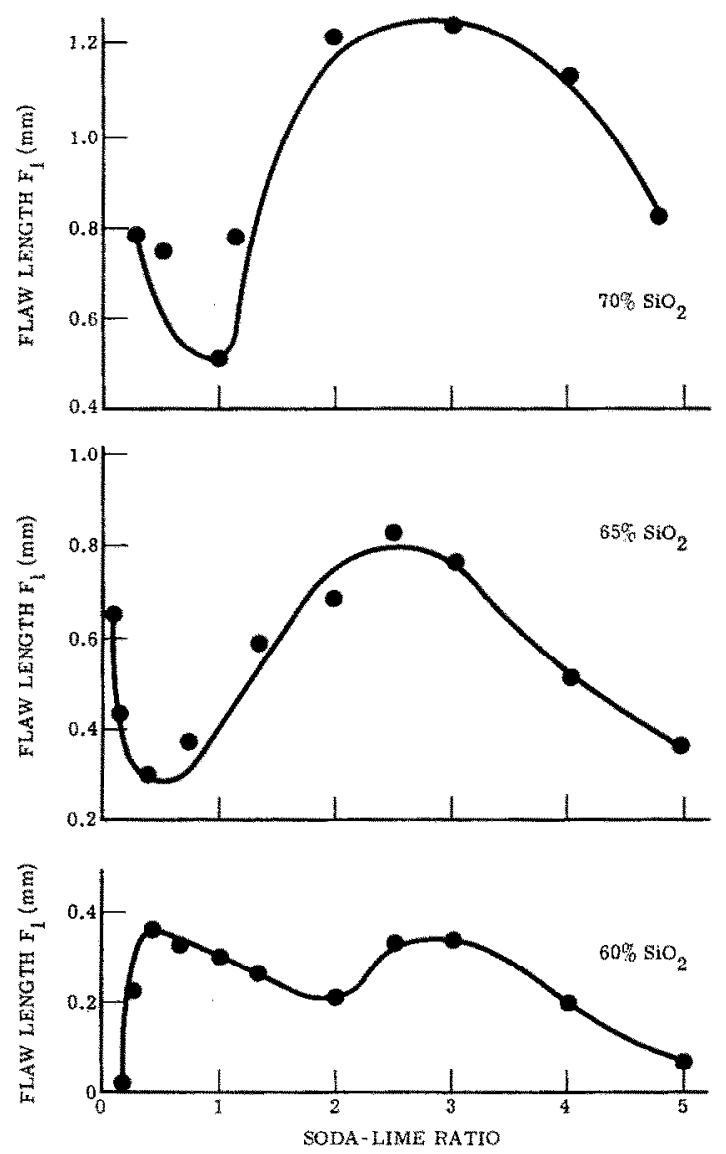

Fic. 2. Flaw length variations in three component systems with different silica bases.

The largest values of $N_{b}$ are found in the 60 per cent $\mathrm{SiO}_{2}$ series with high flaw nucleation numbers. It is also interesting to note that the spiral formations occurred in the 60 per cent $\mathrm{SiO}_{2}$ series with soda-lime ratios from 0.5 to $2 \cdot 0$; also, in this same range, the $F_{n}$ values are very large and the rigidity of the network is high.

The base glass chosen from this group to be used for the network modifying substitutions was the 70 per cent $\mathrm{SiO}_{2}$ glass with a soda-lime ratio of 1.14 . It may be seen in Fig. 2 that this glass is located on a steep portion of the flaw length curve. It appears that changes in the network would be readily detected by marked variations in flaw lengths. This glass is also within the range of composition used in numerous commercial soda-lime-silica systems. The calculated weight percent composition of this base glass is 70 per cent $\mathrm{SiO}_{2}, 16$ per cent $\mathrm{Na}_{2} \mathrm{O}$ and 14 per cent $\mathrm{CaO}$.

\section{A. Network modifying cations}

The majority of the substitutional series were prepared with oxides of the network modifying cations. Oxides of the di- and trivalent cations were substituted for $\mathrm{CaO}$ and the univalent ions for $\mathrm{Na}_{2} \mathrm{O}$. The $\mathrm{Fe}_{2} \mathrm{O}_{3}$ and $\mathrm{CuO}$ additions were in small quantities and were added without substitution for an oxide. Cations in this group are of intermediate sizes and field strengths $\left(Z / r^{2}\right)$ and appeared to produce similar changes in the flaw parameters as they were added into the network. However, in this series of network modifiers, it was found that the effects could be further divided into two subgroups consisting of ions of "medium" " and "low" field strengths.

1. Ions of "medium" field strength. The ions in this subgroup are listed below along with the field strengths as calculated from the data of Pauling, (5) where $r$ is the crystal radius of the ion and $Z$ its charge.

$\begin{array}{ll}\text { Ion } & Z / r^{2} \\ \mathrm{Sn}^{2+} & 3 \cdot 06 \\ \mathrm{Sb}^{3+} & 6 \cdot 52 \\ \mathrm{~Pb}^{2+} & 2 \cdot 53 \\ \mathrm{Bi}^{3+} & 5 \cdot 46 \\ \mathrm{Zn}^{2+} & 3 \cdot 63 \\ \mathrm{Cu}^{2+} & 3 \cdot 06 \\ \mathrm{Li}^{1+} & 2 \cdot 78 \\ \mathrm{Fe}^{3+} & 7 \cdot 32\end{array}$

Because of similar chemical properties, tin and antimony as well as lead and bismuth may be compared as pairs. The flaw parameters for lead and bismuth were previously given; ${ }^{(3)}$ however, their curves are included since they fit into this group of ions.

The similarities in the effects of these ion pairs are shown in Figs 3 and 4 where both the flaw lengths and flaw numbers are plotted on the same 
Table 1. Flaw measurements in three component systems with varying silica bases

$R=$ Soda/Lime ratio

\begin{tabular}{|c|c|c|c|c|}
\hline Series & $\boldsymbol{R}$ & $F_{\mathbf{Z}}$ & $F_{n}$ & $N_{b}$ \\
\hline $60 \% \mathrm{SiO}_{2}$ & $\begin{array}{l}0 \cdot 20 \\
0 \cdot 33 \\
0 \cdot 40 \\
0.50 \\
0 \cdot 66 \\
0 \cdot 80 \\
1.00 \\
1 \cdot 33 \\
2 \cdot 00 \\
2 \cdot 50 \\
3.00 \\
4.00 \\
5.00\end{array}$ & $\begin{array}{l}0.000 \\
0.230 \\
0 \cdot 366 \\
0.333 \\
0.314 \\
0 \cdot 314 \\
0.298 \\
0 \cdot 263 \\
0.204 \\
0 \cdot 336 \\
0.342 \\
0 \cdot 200 \\
0.068\end{array}$ & $\begin{array}{r}0.00 \\
1.84 \\
2.82 \\
10 \cdot 72 \\
12.00 \\
10 \cdot 20 \\
10 \cdot 78 \\
8.00 \\
11.65 \\
1.61 \\
2.10 \\
3.81 \\
2.30\end{array}$ & $\begin{array}{l}0.00 \\
0.42 \\
1.03 \\
3.58 \\
3.86 \\
3.20 \\
3 \cdot 21 \\
2 \cdot 10 \\
2.38 \\
0.54 \\
0 \cdot 72 \\
0 \cdot 76 \\
0 \cdot 16\end{array}$ \\
\hline $65 \% \mathrm{SiO}_{2}$ & $\begin{array}{l}0 \cdot 13 \\
0 \cdot 17 \\
0 \cdot 40 \\
0 \cdot 75 \\
1.33 \\
2 \cdot 00 \\
2 \cdot 50 \\
3 \cdot 00 \\
4 \cdot 00 \\
5 \cdot 00\end{array}$ & $\begin{array}{l}0.658 \\
0.439 \\
0.300 \\
0.375 \\
0.590 \\
0.686 \\
0.825 \\
0.763 \\
0.511 \\
0.362\end{array}$ & $\begin{array}{l}1.52 \\
1.18 \\
1.42 \\
0.68 \\
0.94 \\
1.10 \\
1.42 \\
0.84 \\
0.76 \\
0.55\end{array}$ & $\begin{array}{l}1.02 \\
0.52 \\
0.43 \\
0.26 \\
0.55 \\
0.76 \\
1.17 \\
0.64 \\
0.39 \\
0.20\end{array}$ \\
\hline $70 \% \mathrm{SiO}_{2}$ & $\begin{array}{l}0 \cdot 33 \\
0 \cdot 50 \\
1 \cdot 00 \\
1 \cdot 14 \\
2 \cdot 00 \\
3 \cdot 00 \\
4 \cdot 00 \\
5 \cdot 00\end{array}$ & $\begin{array}{l}0.782 \\
0 \cdot 745 \\
0.516 \\
0.792 \\
1 \cdot 213 \\
1 \cdot 244 \\
1.113 \\
0.815\end{array}$ & $\begin{array}{l}0.70 \\
0.53 \\
0 \cdot 71 \\
0 \cdot 76 \\
0.50 \\
0 \cdot 40 \\
0 \cdot 24 \\
0.12\end{array}$ & $\begin{array}{l}0.55 \\
0.39 \\
0.37 \\
0.60 \\
0.61 \\
0.50 \\
0.27 \\
0.10\end{array}$ \\
\hline
\end{tabular}

curve as a function of the moles of oxide substituted. It should be noted that a different scale (on the right in Figs 3 and 4 ) was used for the $F_{n}$ values due to the pronounced increase in flaw number with moles of oxide added. This method of plotting demonstrates in a direct manner the changes in flaw parameters with moles of oxide substituted into the network of the base glass. As these ions were substituted, there was a general increase in flaw length to a maximum point, followed in subsequent additions by a rapid decrease. In Figs 3 and 4 the maximum $F_{l}$ value and more rapid increase in $\boldsymbol{F}_{n}$ occurs with fewer moles in the case of the ions with the higher field strengths. For example, bismuth oxide of high field strength produces a maximum with fewer moles than lead oxide of low field strength and antimony oxide produces a maximum with fewer moles than tin oxide. The general changes in the flaw parameter curves are similar in these two groups. The significance of the maximum point in the $F_{l}$ curves in Figs 3 and 4 will be discussed presently. The remaining members in this subgroup also produced similar changes in flaw parameters as may be seen by examining the data summarized in 'Table 2 (the zinc oxide curve is given later). 

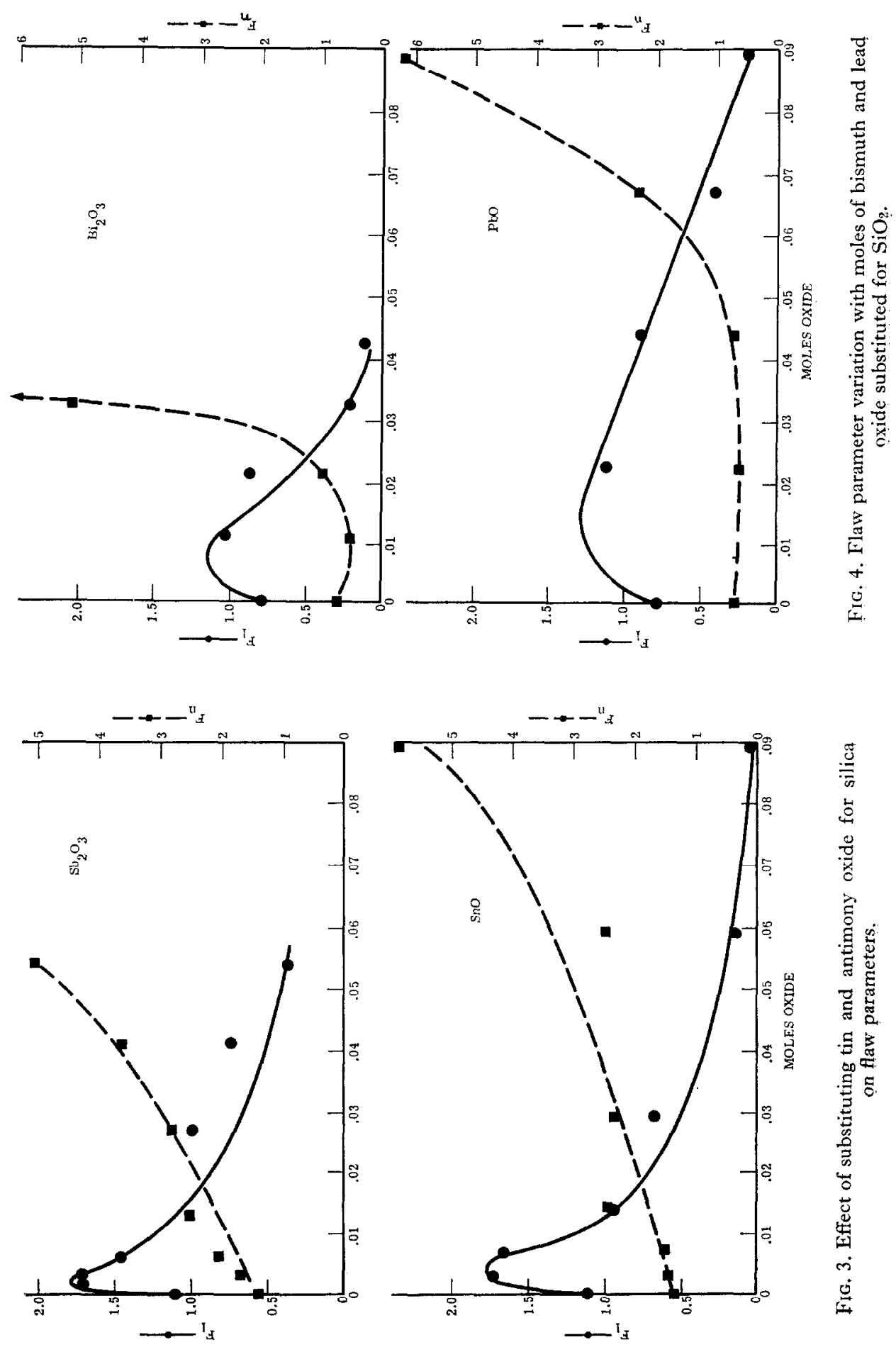
Table 2. Flaw parameters in series containing ions of "medium" field strengths

\begin{tabular}{|c|c|c|c|c|}
\hline Series & Moles oxide & $F_{l}$ & $F_{n}$ & $N_{b}$ \\
\hline $\mathrm{Li}_{2} \mathrm{O}$ & $\begin{array}{l}0.000 \\
0.044 \\
0.174 \\
0.349 \\
0.523 \\
0.610\end{array}$ & $\begin{array}{l}0.792 \\
0.933 \\
0.550 \\
0.397 \\
0.318 \\
0.187\end{array}$ & $\begin{array}{l}0.76 \\
1.32 \\
1.48 \\
2 \cdot 10 \\
2.96 \\
5 \cdot 18\end{array}$ & $\begin{array}{l}0 \cdot 60 \\
1 \cdot 23 \\
0 \cdot 81 \\
0 \cdot 83 \\
0 \cdot 94 \\
0.97\end{array}$ \\
\hline $\mathrm{CuO}$ & $\begin{array}{l}0 \cdot 0000 \\
0 \cdot 0015 \\
0 \cdot 0030 \\
0.0058 \\
0 \cdot 0121 \\
0.0241 \\
0.0390\end{array}$ & $\begin{array}{l}1 \cdot 108 \\
1 \cdot 818 \\
1.629 \\
1 \cdot 529 \\
1.601 \\
1 \cdot 256 \\
1 \cdot 178\end{array}$ & $\begin{array}{l}1 \cdot 39 \\
1 \cdot 30 \\
1 \cdot 61 \\
1 \cdot 71 \\
1 \cdot 90 \\
2 \cdot 22 \\
2 \cdot 39\end{array}$ & $\begin{array}{l}1 \cdot 54 \\
2 \cdot 36 \\
2 \cdot 62 \\
2 \cdot 62 \\
3 \cdot 04 \\
2 \cdot 79 \\
2 \cdot 81\end{array}$ \\
\hline $\mathrm{Fe}_{2} \mathrm{O}_{3}$ & $\begin{array}{l}0.00000 \\
0.00019 \\
0.00038 \\
0.00063 \\
0 \cdot 00125 \\
0 \cdot 00250 \\
0.00501 \\
0.01002 \\
0.02003\end{array}$ & $\begin{array}{l}0.792 \\
1.058 \\
1.283 \\
1.286 \\
1.543 \\
1.457 \\
1.622 \\
0.741 \\
0.580\end{array}$ & $\begin{array}{l}0.76 \\
0 \cdot 66 \\
0 \cdot 78 \\
0 \cdot 82 \\
0 \cdot 83 \\
0 \cdot 90 \\
1 \cdot 30 \\
1 \cdot 83 \\
2 \cdot 32\end{array}$ & $\begin{array}{l}0 \cdot 60 \\
0 \cdot 70 \\
1 \cdot 00 \\
1 \cdot 06 \\
1 \cdot 28 \\
1 \cdot 31 \\
2 \cdot 11 \\
1 \cdot 36 \\
1 \cdot 35\end{array}$ \\
\hline
\end{tabular}

Again each ion produced changes of slightly different magnitude.

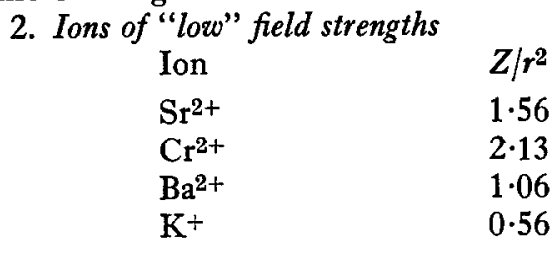

The flaw parameter data for $\mathrm{ZnO}$ from subgroup 1 and SrO from the "low" field strength group are plotted in Fig. 5 to demonstrate the differences between ions in these two series. In general, the flaw length changes in the subgroup 2 ions are similar to those in the "medium" field strength subgroup. With the "low" field strength ions, a greater amount of oxide is necessary to reach the maximum in the flaw length curve. Also, the $F_{n}$ curves in Fig. 5 disclose a general decrease in flaw number in the SrO glasses in contrast with the pronounced increase in flaw number in the $\mathrm{ZnO}$ series with the ions of "medium" field strength. The flaw data for $\mathrm{BaO}, \mathrm{CdO}$ and $\mathrm{K}_{2} \mathrm{O}$ are given in Table 3 , and these ions also produce effects similar, to the $\mathrm{SrO}$ results; particularly the cadmium oxide with the marked increase in flaw length and decreasing flaw number. The $\mathrm{K}^{+}$and $\mathrm{Ba}^{2+}$ ions both disclose initial increases in flaw number instead of decreases; however, it was felt that they should be included in this subgroup because of the shapes of the flaw length curves. The $\mathrm{K}^{+}$ion behaves almost like a "pure" interstitial as will be pointed out later.

\section{B. Discussion of network modifying effects}

One characteristic feature of the flaw length curves in both the network modifying series is the increase to a maximum value. These general changes are shown schematically in Fig. 6 with $M$ representing the moles of oxide substituted into the network. Conceivably the modifying cations initially fill intersticies in the network. As the holes become filled, the ions may take up lattice positions; however, these internal energy changes are not considered as being a monotonic function of the substituted cation. An example of this non 

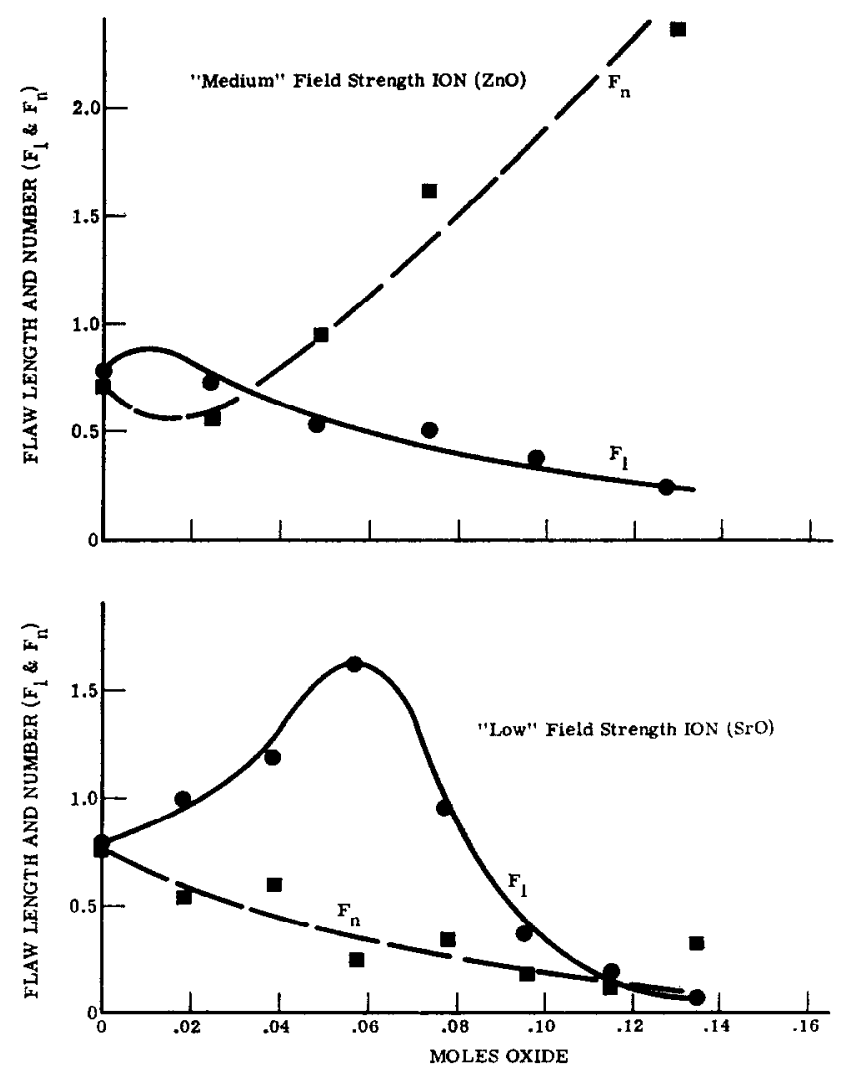

FIG. 5. Variations in flaw parameter curves using net-work modifying ions of "medium" and "low" field strength (substitutions for $\mathrm{CaO}$ ).

uniform change in energy has been given by DIETZEL and WICKERT ${ }^{(6)}$ for a binary silicate. As $\mathrm{Na} 2 \mathrm{O}$ was added to silica the rates of crystallization disclosed maxima and minima. The maximum rates of crystallization occurred in glasses with compositions corresponding to the di- and metasilicates. This type of change in internal structure may occur with varying sodium and calcium ion ratios and could at least partially explain the formation of the maxima and minima observed in the curves in Fig. 2.

In the various series reported here, the cations replace either sodium or calcium (with the exception of a few cases such as bismuth and lead and where small additions were made). Replacement or addition of ions of different size creates lattice defect through misfit and bond distortion. It should be kept in mind, however, that defects are present in the base glass and the substitutions only modify the existing defect structure. As more "misfit" ions are added into the network, the defect concentration increases, until the ions no longer enter as interstitials but instead form stronger ionic or covalent associations in the network. The point of maximum flaw length (at $M^{\prime}$ in Fig. 6) is thought to represent the critical defect concentration or maximum disorder in the system. Also, in Fig. $6, N$ represents the number of ions necessary to produce the maximum disorder.

The free energy $(F)$ of the system is expressed by,

$$
F=\epsilon-T\left(S_{c f}+S_{t h}\right)
$$

where $S_{c f}$ is the configurational entropy produced by $N$ ions creating $n$ defects and $S_{t h}$ the thermal entropy. The defects formed may be combinations 
of both Schottky (cation-anion vacancies) and Frenkel types. In the formation of the Frenkel disorder, the ions (cations more likely than anions) pass from lattice sites into interstitial positions with the formation of vacant lattice sites and there is less volume change as compared with the Schottky type. The Frenkel type of disorder is more likely when the lattice polarization is strong and may, therefore, predominate in these substitutional series.

It is assumed that the thermal entropy $S_{t h}$ will change with the added ions to a lesser degree than the configurational entropy. As pointed out by DEKKER (7) $S_{t h}$ may be considered to be independent of $n / N$. In the case shown here, the introduction of ions with different field strengths increass the disorder and the negative entropy term produces a decrease in the free energy as the ions are initially added and defects are created. Such a decrease would be thermodynamically favorable and defect formation could, therefore, more than compensate for any initial increase in $(\epsilon)$ within the network.

The point $M^{\prime}$, therefore, indicates the maximum $S_{c f}$ influence of randomly distributed defects. The point at $M^{\prime}$ may also represent the maximum randomization of free volume $\left(v_{f}\right)$ defined by Turnbull and $\mathrm{CoHeN}^{(8)}$ as

$$
v_{f}=\bar{v}-v_{0}
$$

where $\bar{v}$ is the specific volume and $v_{0}$ is the van der Waals volume of a molecular region. These authors have suggested that molecular transport may occur by the formation of "voids" during the free volume redistribution. They further point out that the random distribution of this unoccupied volume may occur more readily in the amorphous than in the crystalline phase. Any increase in order would, therefore, tend to decrease the free volume of the system.

The application of an external stress to a system with maximum disorder would allow considerable structural yield and bond readjustment as a result of the free volume redistribution, with the maximum free volume change occurring at the point of maximum defect (void) concentration or at $M^{\prime}$ in the $F_{l}$ curve. A structural yield and free volume redistribution could conceivably produce a slight decrease in the $F_{n}$ curve since more bonds would bend instead of creating slip and nucleating flaws.
Although the $F_{n}$ data shows scatter, this predicted decrease in $F_{n}$ to the point $M^{\prime}$ may be indicated experimentally, specifically in Fig. 4 and in the upper curves in Fig. 5.

With ionic additions $M>M^{\prime}$, both complex ionic and covalent bonds begin to form through chemical association with the added cations. The lattice is, therefore, additionally modified by the formation of more extensively ordered groupings and a consequent reduction in energy $(\epsilon)$. With the formation of more ordered units and stronger bonds the observed decrease in $F_{l}$ (beyond $M^{\prime}$ ) is readily explained; however, along with the formation of ordered groupings, structural yield or bending of bonds would tend to decrease and flaw nucleation under applied stress would increase. 'I'his would explain the rapid increase in $\boldsymbol{F}_{\boldsymbol{n}}$ with additions greater than $M^{\prime}$, as observed in Figs 3 and 4. It appears that in the case of larger ions in the "low" field strength series (subgroup 2) a greater misfit of the ions produces a continuous increase in defect concentration (and free volume), which is not entirely compensated by an increase in order. The bond flexibility increases with creation of the free volume, and $F_{n}$ decreases as shown in the lower curves in Fig. 5 and in Table 3.

In many of the series, the glasses crystallized with additions greater than point $M_{c}$ in Fig. 6 . The kinetic barrier for nucleation may rapidly decrease after the point $M^{\prime}$ and as ordered groupings form, crystal nucleation is promoted.

\section{Interstitial additions}

These same general defect hypotheses may be applied to the effects of interstitials on flaw parameters. The addition of interstitials produces steady increases in $S_{c f}$ with increasing $M$; however, no rapid decrease in energy $(\epsilon)$ occurs. There are no strong bonds or ordered groupings formed between the added ions and the surrounding network. As the structure steadily weakens, both $F_{l}$ and $F_{n}$ increase. Carbon provides an excellent example for the interstitial effect as shown in Fig. 7. The carbon was added in low percentages without substitution for any specific oxide and the glass became smokey in appearance as additions were made. The interstitial or "insoluble" nature of carbon in non-sulfur and non-iron containing glasses is well known and an excellent discussion may be found in WEYL's book on colored glasses. (9) 
W. C. LEVENGOOD

Table 3. Flaw parameters in series containing ions of "low" field strengths

\begin{tabular}{|c|c|c|c|c|}
\hline Series & Moles oxide & $F_{l}$ & $F_{n}$ & $N_{b}$ \\
\hline $\mathrm{K}_{2} \mathrm{O}$ & $\begin{array}{l}0 \cdot 000 \\
0 \cdot 018 \\
0 \cdot 073 \\
0 \cdot 145 \\
0 \cdot 218\end{array}$ & $\begin{array}{l}0.792 \\
0.929 \\
0.942 \\
1.024 \\
1.000\end{array}$ & $\begin{array}{l}0.76 \\
1.07 \\
1.31 \\
1.67 \\
1.60\end{array}$ & $\begin{array}{l}0.60 \\
0.99 \\
1.23 \\
1.71 \\
1.60\end{array}$ \\
\hline $\mathrm{CdO}$ & $\begin{array}{l}0.000 \\
0.016 \\
0.031 \\
0.047 \\
0.062 \\
0.078\end{array}$ & $\begin{array}{l}0.792 \\
1 \cdot 231 \\
1.344 \\
1.639 \\
1.907 \\
0.000\end{array}$ & $\begin{array}{l}0 \cdot 76 \\
0 \cdot 41 \\
0 \cdot 42 \\
0 \cdot 37 \\
0 \cdot 14 \\
0.00\end{array}$ & $\begin{array}{l}0.60 \\
0.50 \\
0.56 \\
0.60 \\
0.27 \\
0.00\end{array}$ \\
\hline $\mathrm{BaO}$ & $\begin{array}{l}0.000 \\
0.006 \\
0.027 \\
0.052 \\
0.078 \\
0.091\end{array}$ & $\begin{array}{l}0.792 \\
1.006 \\
0.849 \\
0.602 \\
0.132 \\
0.068\end{array}$ & $\begin{array}{l}0.76 \\
1.08 \\
1.72 \\
0.63 \\
0.09 \\
0.10\end{array}$ & $\begin{array}{l}0.60 \\
1.09 \\
1.46 \\
0.38 \\
0.12 \\
0.01\end{array}$ \\
\hline
\end{tabular}

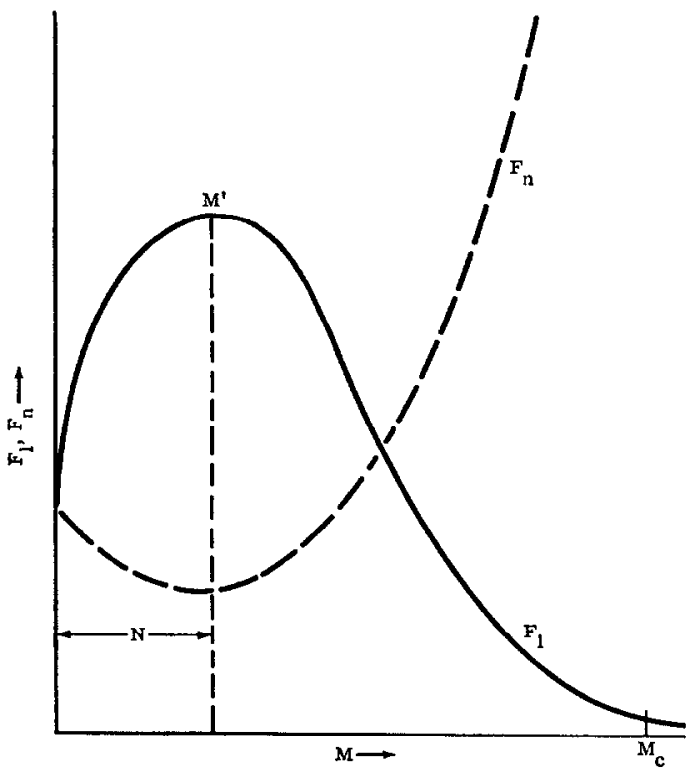

FIG. 6. Schematic diagram showing general effect of network modifying ions on flaw parameters

$\boldsymbol{M}$-moles of oxide substituted

$M^{\prime}$-critical defect concentration; point of maximum disorder.

$N$-number of ions necessary to produce maximum disorder.

$M_{c}$ - point of maximum order; just preceding crystallization.

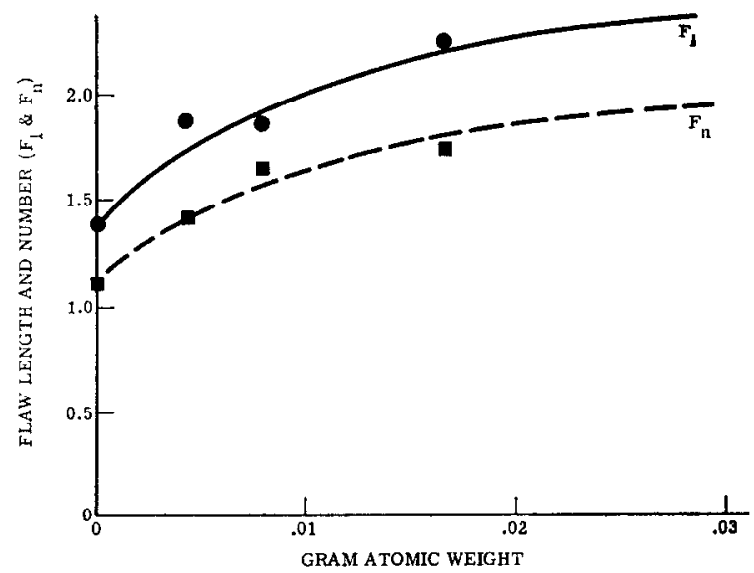

FIG. 7. Influence of carbon on flaw parameters.

In the discussion of the effect of fluorine in glass, Weyl also points out that substitution of the monovalent fluorine ion for divalent oxygen "causes a weakening of the glass structure". This weakening effect of fluorine was confirmed in a series with $\mathrm{NaF}$ substituted for $\mathrm{Na}_{2} \mathrm{O}$. The number of $\mathrm{Na}^{+}$ions was held constant as the additions were made. As shown in Fig. 8 , both $F_{l}$ and $F_{n}$ initially increase as the structure weakens. With additions greater than about 0.25 moles $\mathrm{NaF}$ 
crystallization occurred. The decreasing values of $F_{l}$ and $F_{n}$ at higher additions of $\mathrm{NaF}$ may, therefore, represent a decrease in free energy as ordered groupings precede the visible, fluoride opal color. A maximum is observed in the $F_{1}$ curve as in the case of the modifying cations (point $M^{\prime}$ in Fig. 6); however, because of the pronounced initial increase in both the flaw parameters, this ion is considered as having an interstitial effect.

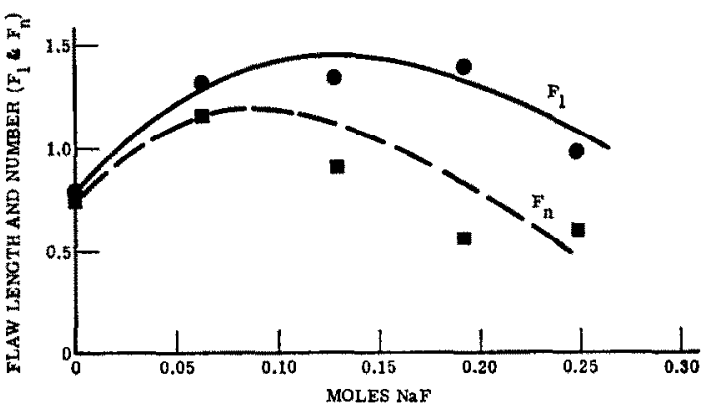

Frc. 8. Effect of the fluorine ion on flaw formation.

It is also problematical whether the results from the $\mathrm{K}^{+}$series should also be placed in the interstitial column. As a result of its low field strength potassium appears to possess both modifying and interstitial properties (see data in Section A).

\section{Network former effects}

The so-called network formers have very high field strengths and replace the strong siliconoxygen bonds. Network formers may produce a greater effect on internal energy $(e)$ than on disorder $\left(S_{c f}\right)$. Although the effects are more difficult to predict, one might expect less change in $F_{l}$ with added ions or perhaps even a decrease in some cases if the bond strength is increased. Ordered groupings through chemical association might also be expected to be less than in the case of the modifying ions and consequently $F_{n}$ would remain unchanged or perhaps decrease. The various ions which were substituted for silica are as follows

$\begin{array}{ll}\text { Ion } & \left(Z / r^{2}\right) \\ \mathrm{P}^{5+} & 41 \cdot 67 \\ \mathrm{Al}^{3+} & 12 \cdot 00 \\ \mathrm{~B}^{3+} & 75 \cdot 00\end{array}$

The substitution of both $\mathrm{P}_{2} \mathrm{O}_{5}$ and $\mathrm{Al}_{2} \mathrm{O}_{3}$ were found to produce the effects on the flaw parameters as predicted. As shown in Table 4, both the flaw lengths and number decrease with the addition of these ions with high field strengths. Additions of $\mathrm{Al}_{2} \mathrm{O}_{3}$ greater than around 0.05 moles produced immiscibility and reliable flaw measurements could not be obtained.

In the case of $\mathrm{B}_{2} \mathrm{O}_{3}$, the results were much

Table 4. Flaw parameters in series containing ions with high field strengths

\begin{tabular}{lcccc}
\hline \hline \multirow{2}{*}{ Series } & Moles oxide & $F_{l}$ & $F_{n}$ & $N_{b}$ \\
\hline $\mathrm{Al}_{2} \mathrm{O}_{3}$ & 0.000 & 0.792 & 0.76 & 0.60 \\
& 0.010 & 0.306 & 0.66 & 0.20 \\
& 0.049 & 0.284 & 0.63 & 0.18 \\
$\mathrm{P}_{2} \mathrm{O}_{5}$ & & & & \\
& 0.000 & 1.108 & 1.39 & 1.54 \\
& 0.014 & 0.784 & 1.23 & 0.96 \\
& 0.035 & 0.594 & 0.83 & 0.49 \\
$\mathrm{~B}_{2} \mathrm{O}_{3}$ & & & & \\
& 0.000 & 0.792 & 0.76 & 0.60 \\
& 0.072 & 0.917 & 0.42 & 0.38 \\
& 0.144 & 0.971 & 0.38 & 0.37 \\
& 0.215 & 1.114 & 0.49 & 0.55 \\
& 0.287 & 1.023 & 0.54 & 0.55 \\
& 0.359 & 1.006 & 0.56 & 0.56 \\
& 0.431 & 0.990 & 0.62 & 0.61 \\
& 0.502 & 0.853 & 0.78 & 0.67 \\
& 0.574 & 0.948 & 0.28 & 0.27 \\
& 0.646 & 0.681 & 0.40 & 0.27 \\
\hline
\end{tabular}


more difficult to explain, even on a qualitative basis. The flaw results in Table 4 show the more erratic behavior of the boron ion. $\mathrm{A}_{2} \mathrm{~B}_{3}$ glass is however noted for its complex changes in structure. As the $\mathrm{B}^{3+}$ ion enters the network, vacancy defects may be created as it replaces the silicon-oxygen bonds. This would explain the initial increase in $F_{l}$ and decrease in $F_{n}$ as was observed in the case of the large modifying ions. The difference in charge and the high field strength of the $\mathrm{B}^{3+}$ ion may account for complex structural groupings in the network. For example, WeYL and MARBoE ${ }^{(10)}$ point out that vitreous $\mathrm{B}_{2} \mathrm{O}_{3}$ may consist of molecules which have widely different molecular weights and odd shapes. Additional study of defect structures in systems containing the $\mathrm{B}^{3+}$ ion might shed more light on the influence of this ion on the structural changes.

\section{E. Anomalous behavior of $\mathrm{MgO}$}

The effects produced by the substitution of $\mathrm{MgO}$ for $\mathrm{CaO}$ were difficult to categorize. In general, Fig. 9 indicates that the $\mathrm{Mg}^{2+}$ ion

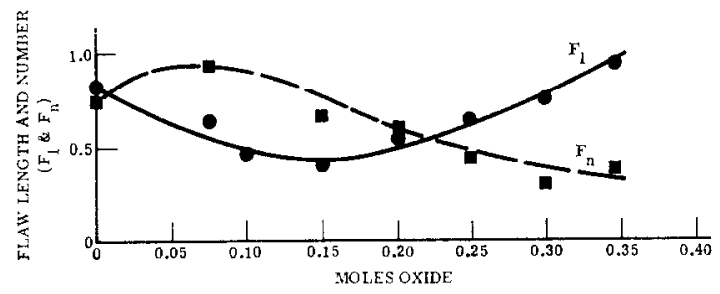

Fig. 9. Flaw variations in glass with $\mathrm{MgO}$ substituted for $\mathrm{CaO}$.

$\left(Z / r^{2}=4 \cdot 76\right)$ produces effects similar to the network formers with an initial decrease in $F_{l}$ and little change in $F_{n}$. An increase in $F_{l}$ occurs, however, as the point of complete substitution is approached. The $F_{n}$ values continue to decrease and the final structure appears to be weak with low brittleness or high bond flexibility. The anomalous behavior of the $\mathrm{Mg}^{2+}$ ion has also been discussed by Petzold, WishManN and Kamptz ${ }^{(11)}$ in their studies of the microhardness of glass. It was suggested that magnesium may, as the result of oxygen coordination changes, take either network modifying or network forming positions.

\section{F. Ionic field strength of lattice modifying ions}

It was previously suggested in Section B that the number $(N)$ of lattice modifying ions producing the maximum disorder (point $M^{\prime}$ in Fig. 6) is related to the field strength of the ions. This number $(N)$ was determined (as $\mathrm{g}$ at. wt) in all of the series showing the maximum $\left(M^{\prime}\right)$ and Fig. 10 shows the results of plotting with the field strength of the corresponding ion. The number of ions producing the maximum disorder change by three orders of magnitude over this limited range of field strength. The scatter of the points in Fig. 10 in many cases, is due to uncertainty in locating the point $M^{\prime}$. In most series all the glasses were made up before the flaw parameters were determined and it was necessary to extrapolate between missing data points at low concentrations. The results in Fig. 10 indicate the influence of ionic field strength of the lattice modifying cations on the flaw parameters. These findings are also in accord with the suggestions of WEYL and MARBoE, (12) who also stressed the importance of ionic configuration in determining defect structures in glass.

\section{DISCUSSION OF RESULTS}

It appeared pertinent to compare the flaw parameter results with recent studies by LoEwENSTEIN (13) of glasses possessing high Young's moduli. In many cases he substituted the same ions as used in the series reported in the preceding Sections. The compositions of the glasses used by Loewenstein were not identical with those reported here; therefore, any comparisons will by necessity be qualitative in nature.

The flaw parameter most closely related to Young's modulus is the number of flaws nucleated per length of trace line $\left(F_{n}\right)$. A high Young's modulus $(E)$ indicates a rigid structure with little yield under applied stress. As discussed in Section III the $F_{n}$ value would also be high in a glass with high $E$; this proposed relation might be expressed by,

$$
E \approx C F_{n}
$$

where $C$ is a proportionality constant. There are some excellent qualitative agreements between Loewenstein's work and the changes in the $F_{n}$ parameter as various ions were substituted. For example, it is pointed out that the network formers 
such as $\mathrm{Si}, \mathrm{B}, \mathrm{P}$ and $\mathrm{Al}$ lower the Young's modulus. Data in Table 1 show the decrease in $F_{n}$ with increasing $\mathrm{SiO}_{2}$, and Table 4 demonstrates decreasing $F_{n}$ for $\mathrm{B}, \mathrm{P}$ and $\mathrm{Al}$ catione (the boron values are more erratic as was pointed out). The network formers substantiate the relation indicated in equation (9).
Potassium is an apparent exception in the comparison of these two different studies. It was reported by Loewenstein that $\mathrm{K}^{+}$decreased $E$ whereas the $F_{n}$ value increased with substitution of potassium for sodium. The substitution of $\mathrm{Li}_{2}$ for $\mathrm{Na}_{2} \mathrm{O}$ produced marked increase in $F_{n}$ and an increase in $E$ was also reported for this substitu-

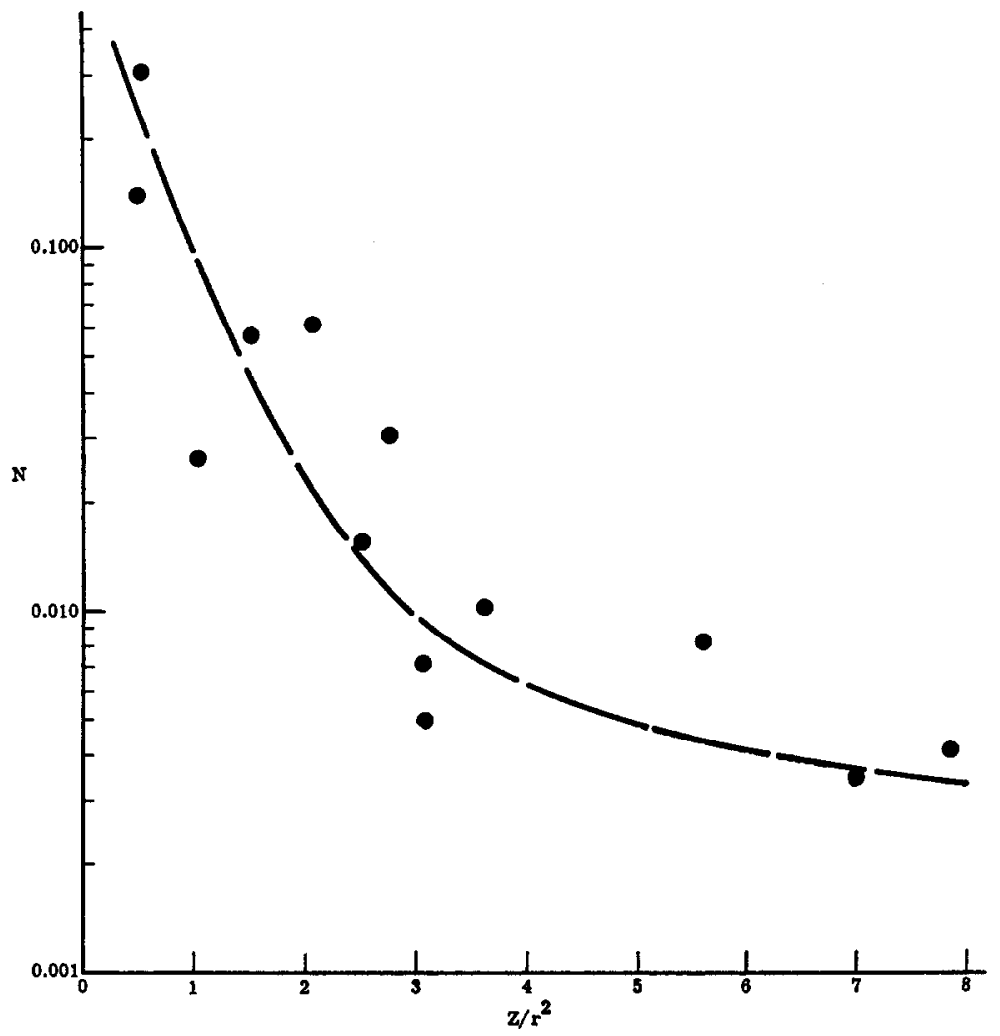

Fig. 10. Relation between the number of ions producing maximum disorder and ionic field strength. ( $N$ is given as gram atomic weight of ions in the glass.)

Loewenstein also states that "ions which do not enter the network yet increase the Young's modulus appreciably possess a low solubility in the base glass". An excellent example of this is shown by carbon which increases $F_{n}$ but does not significantly enter the network. Large ions, such as $\mathrm{Sr}$ and $\mathrm{Ba}$ were reported to lower $E$ and this also appears to be generally in agreement with the present findings. An examination of the flaw parameter curves show that initial additions of the modifying ions tend to produce decreases in $F_{n}$. tion. In this rather superficial comparison it is indicated that there is a relation between Young's modulus and the $F_{n}$ parameter. By comparing $E$ and $F_{n}$ on the same glasses, the relationship could be more rigorously determined than given by equation (9).

VI. SUMMARY

Defects created under controlled loading conditions were utilized in an investigation of structural variations in glasses produced by ionic 
substitutions. Detailed changes in the flaw parameters were shown to be related to the manner in which an ion entered the network; that is as a modifier, interstitial or network former.

Ions of intermediate field strength, or the network modifiers, produced an initial disorder in the structure and an increase in the flaw length values. With increasing ions substituted, a maximum occurred in the flaw length curve. With additions beyond this maximum the flaw lengths rapidly decreased and the flaw number disclosed a marked increase. These variations in flaw parameters were attributed to free energy changes as the ions were substituted. Vacancies and interstitial defects are believed to be formed as ions were initially added and these promoted a free volume redistribution of voids, which was most pronounced at the maximum in the flaw length curves. With additions beyond this maximum point, the ions formed chemical associations and the internal order increased. The formation of ordered groupings increased the bond strength and the flaw length decreased. The ordered groupings also increased the brittleness and, in most cases, the flaw number $\left(F_{n}\right)$ increased beyond the maximum.

These same general defect hypotheses were used to explain interstitial and network former effects. In the case of interstitial additions, such as carbon, no strong associations developed and the flaw lengths and number increased continuously with increasing concentration. The interstitials were ions of low field strengths. The network formers or ions with high-field strengths replaced $\mathrm{Si}$ in the network and the bond strengths generally increased with resulting decreases in flaw lengths and number.

Superficial comparisons were also made between the studies reported here and work by Loewenstein on ionic substitutions effecting Young's modulus of glass. These qualitative comparisons showed that as predicted (in Section III) the $F_{n}$ values are a measure of the brittleness of the glass network. In general ions causing an increase in $E$ also disclosed increases in $F_{n}$ and those producing a decrease in $E$ disclosed decreasing $F_{n}$ values. By determining these parameters on the same glass, the relationship between $E$ and $F_{n}$ (and $F_{l}$ ) could be be more precisely determined.

Acknowledgements-The author would like to express his appreciation to Mr. William Wolfe, at the University of Michigan, for his very helpful comments and to Mr. R. A. Gaiser, director of research, Ball Brothers Research Corp., Muncie, Indiana for allowing portions of the experimental results to be released for publication.

\section{REFERENCES}

1. Levengood W. C., J. appl. Phys. 30, 378-386 (1959).

2. Levengood W. C. and Vong 'T. S., J. appl. Phys. 31, 1416-1421 (1960).

3. Levengood W. C., J. appl. Phys. 32, 2525-2533 (1961).

4. Grax 'T. J., The Defect Solid State, Interscience, New York (1957).

5. Pauling L., The Nature of the Chemical Bond, Cornell University Press, New York (1960).

6. Dietzel A. and Wickert H., Glastech. Ber. 29, 1 (1956).

7. Dekker A. J., Solid State Physics, Prentice Hall, Englewood Cliffs, N.J. (1957).

8. Turnbull D. and Cohen M. H., J. chem. Phys. 34, 120-125 (1961).

9. WeyL W. A., Coloured Glasses, The Society of Glass 'Technology, Sheffield, England (1951).

10. Weyl W. A. and Marboe E. C., Glass Ind. 41, 625 (1960).

11. Petzold A., Wishmann F. G. and Kamptz H., Glastech. Ber. 34, 56-71 (1961).

12. Weyl W. A. and Marboe E. C., J. Soc. Glass Tech. 43, 417 (1959).

13. Loewenstein K. L., Phys. Chem. Glasses, 2, 69-82 (1961). 